\title{
AN ALTERNATIVE TREATMENT TO ELECTROPOLISHING: CHEMICAL POLISHING
}

\author{
Harleen Kaur and Prabhjot Kaur \\ Department of Applied Sciences and Humanities, Shaheed Bhagat Singh State Technical Campus, \\ Ferozepur (Punjab)-152004, India Author for correspondence \\ E-mail: harleen.sodhi5@gmail.com, prabhjotsandhu1@gmail.com
}

\begin{abstract}
:
Anodic alumina template containing hexagonally ordered nanopores has been widely used over the last decade for the development of numerous functional nanostructures such as nanoscale sensors, computing networks, optoelectronic devices, thin film micro circuits, ultra filtration membranes, keying layers for organic adhesives to hard, wear and corrosion resistant layers and memories. The long range pore order requires the starting aluminum surface to be extremely smooth. Electropolishing is the most commonly used method for surface planarization prior to anodization. While prevalent, this method has several limitations in terms of throughput, polishing area and requirement of special experimental setups. In this work, we report a new treatment called-chemical polishing which offers a viable, simpler, safer and faster alternative to electropolishing.
\end{abstract}

Keywords: Electro polishing, Chemical polishing, Surface roughness tester.

\section{Council for Innovative Research}

Peer Review Research Publishing System

Journal: Journal of Advances in Chemistry

Vol 1, No. 1

editor@cirworld.com

www.cirworld.com, member.cirworld.com

1 | P a g e

June 15,2013 


\section{Introduction}

The electropolishing system smoothens, polishes, deburrs, cleans and streamlines the microscopic surface of a metal object. The process selectively removes high points on metal surfaces, giving the surface a high luster. As a result, the surface of the metal is microscopically featureless, with not even the smallest speck of a tom surface remaining. In literature, electropolishing is the most commonly used surface planarization technique [1-6]. Several electrolytes such as perchloric acid-ethanol mixture [7-11] and a mixture of phosphoric acid, sulphuric acid, water (or chromic oxide) $[12,13]$ have also been reported. Interestingly, perchloric acid is a common ingredient in most cases and produces better smoothness compared to the solutions that do not contain perchloric acid [14].

While popular, electropolishing (with a solution that contains perchloric acid) comes with several practical disadvantages all of which originate from the fact that hot perchloric acid is a hazardous chemical which requires special handling. For example, during electropolishing the electrolyte gets heated and hot perchloric acid is well known for its notoriously strong oxidizing properties and can also become unstable. This requires continuous cooling of the electrolyte during polishing. Even storage of these electrolytes require special perchloric acid fume hood with self-contained wash-down units to inhibit formation of metallic per sschlorate crystals on the walls of the fume hood and the exhaust ductwork. These crystals are highly unstable and shock-sensitive and may ignite or detonate under certain conditions. Using home-built electropolishing setups it is possible to simultaneously electropolish multiple aluminum substrates in parallel in an electrolyte, but again this generates larger amount of heat and thus, requiring highly efficient heat removal from the system. Thus an alternative fast polishing technique that will avoid these disadvantages and at the same time produce $\mathrm{AAO}$ templates of comparable quality is highly desirable.

In this work, we draw renewed attention on the comparison of chemical polishing and electropolishing on the aluminium template to optimize an important parameter, time has been evaluated for obtaining least roughness and highly brightness surface by using roughness tester. Recently, $\mathrm{Yu}$ et al. [15] investigated the influences of electropolishing variables on the surface roughness of industrially pure aluminium using factorial design. However, there is little reported research on using roughness tester to evaluate the aluminium surface. This method has been rarely used in the development of nanostructures even though it allows parallel processing of large number of aluminum substrates with arbitrary areas and not necessarily requires perchloric acid. In this report, the chemical polishing process is characterized by the use of a mixture of nitric and phosphoric acid. The results and discussions will be presented in Section 3.

\section{Experimental}

\subsection{Sample preparation}

Aluminum specimens were cut from high purity unpolished aluminum sheet $(99.997 \%), 0.33 \mathrm{~mm}$ thick, into rectangular shape having width $2 \mathrm{~cm}$ and $16 \mathrm{~cm}$ length. Larger area samples $(10 \mathrm{~cm} \times 10 \mathrm{~cm})$ were also studied and no significant difference has been observed. After cutting, samples were first degreased in ethanol for $200 \mathrm{~s}$, then washed in deionised water and air-dried. These samples were then annealed at $673 \mathrm{~K}$ for 2 hrs in temperature controlled silicon carbide tube furnace and then cooled for $22 \mathrm{hrs}$. Annealing is necessary to improve the homogeneity of the aluminium substrate for well ordered pore growth, by increasing grain size, thus minimizing grain boundary area which is considered as a defect and eliminating residual stresses obtained as a result of rolling. These unpolished samples have been subjected to the proposed electropolishing and chemical polishing step.

\subsection{Electropolishing}

Electropolishing was carried out under dc biased Potentiostat/Galvanostat at 15V. During electropolishing the sample was used as the working electrode with an exposed area of $1 \times 1 \mathrm{~cm}$. The electropolishing solution was composed of mixture of perchloric acid and ethanol (analytical reagent) with volume ratios of 1:5. The temperature was maintained at $100 \mathrm{C}$ by thermostated water circulation through the electrochemical cell and the electropolishing time was varied from $3 \mathrm{~min}$ to $9 \mathrm{~min}$. Under these conditions, electropolishing current density is $\sim 0.04 \mathrm{~A} / \mathrm{cm}$ which results in evolution of considerable amount of heat.

\subsection{Chemical polishing}

Chemical polishing was done by the mixture consists of 15 parts of $68 \%$ nitric acid and 85 parts of $85 \%$ phosphoric acid. This method completely avoids perchloric acid and the related hazards. The etching is performed for various durations (3-9 min for the temperature range $10^{\circ} \mathrm{C}$. After etching, the samples are neutralized in $1 \mathrm{M}$ sodium hydroxide for $20 \mathrm{~min}$. Specimens obtained from both the cases then be compared to find out optimum condition of crucial parameter time. 


\section{Result and discussion}

In our work we demonstrate after chemical polishing, the entire surface is shiny and mirror-like and appears almost identical to the electropolished surface after 4 minutes $30 \mathrm{sec}$. Thus the proposed method is a viable, simpler, safer, faster alternative for fabrication of good quality AAO templates. Surprisingly, under certain polishing conditions we observed pitting in case of electropolishing and dissolution of metal surface in case of chemical polishing.

To characterize and compare the surface roughnesses under different polishing conditions, we use portable surface roughness tester.

\begin{tabular}{|c|c|c|c|}
\hline S.NO. & $\begin{array}{l}\text { Time } \\
\text { (in minutes) }\end{array}$ & $\begin{array}{l}\text { Roughness } \\
\text { (in } \mu \mathrm{m} \text { ) in case of } \\
\text { electro polishing }\end{array}$ & $\begin{array}{l}\text { Roughness } \\
\text { (in } \mu \mathrm{m} \text { ) in case of } \\
\text { chemical polishing }\end{array}$ \\
\hline 1) & 3minutes & 0.63 & 1.00 \\
\hline 2) & $\begin{array}{l}\text { 4minutes } \\
\text { seconds }\end{array}$ & 1.59 & 1.07 \\
\hline 3) & 6 minutes & 1.67 & 1.36 \\
\hline 4) & $\begin{array}{l}\text { 7minutes } \\
\text { seconds }\end{array}$ & 1.44 & 1.31 \\
\hline 5) & 9 minutes & 1.44 & 1.31 \\
\hline
\end{tabular}

Table 1. Effect of time on surface roughness with electropolishing

It has been observed from the above results that for electropolishing and in chemical polishing 3 minutes are sufficient for obtaining smooth and lustrous surface. On increasing the time roughness first increases (up to 6 minutes) and then decreases. After a certain time it becomes constant.

\section{Conclusions}

In these studies, longer electropolishing time did not show significant improvement in surface roughness. Although, electropolishing is very successful method to obtain better surface, yet chemical polishing method reported here will still remain a simpler, safer and faster alternative. Thus chemical polishing approach is more appealing for fast fabrication of high quality AAO samples in a simple and less hazardous fashion.

\section{References}

[1] H. Masuda, M. Satoh, Japanese Journal of Applied Physics., 35, (1996), L126.

[2] K. Yasui, K. Nishio, H. Nunokawa, H. Masuda, Journal of Vacuum Science and

Technology B., 23, (2005), L9.

[3] H. Masuda, H. Yamada, M. Satoh, H. Asoh, M. Nakao, T. Tamamura, Applied Physics

Letters., 71, (1997), 2770.

[4] I. Mikulskas, S. Juodkazis, R. Tomasiunas, J. Dumas, Advanced Materials., 13, (2001), 1574.

[5] S. Bandyopadhyay, A. Miller, H. Chang, G. Banerjee, V. Yuzhakov, D.-F. Yue, R. Ricker, S. Jones, J. Eastman, E. Baugher, M. Chandrasekhar, Nanotechnology., 7, (1996), 360.

[6] C. Liu, A. Datta, Y. Wang, Applied Physics Letters., 78, (2001), 120.

[7] R. Ricker, A. Miller, D.-F. Yue, G. Banerjee, S. Bandyopadhyay, Journal of Electronic

Materials., 25, (1996), 1585.

[8] L. Ba, W. Li, Journal of Physics. D. Applied Physics., 33, (2000), 2527. 
[9] W. Lee, R. Ji, U. Gösele, K. Nielsch, Nature Materials., 5, (2006), 741.

[10] M. Tian, S. Xu, J. Wang, N. Kumar, E. Wertz, Q. Li, P. Campbell, M. Chan, T. Mallouk, Nano Letters., 5, (2005), 697.

[11] N. Tasaltin, S. Ozturk, H. Yuzer, Z. Ozturk, Journal of Optoelectronic and Biomedical Materials 1., (2009), 79.

[12] A. Vazquez, R. Carrera, E. Arce, N. Castillo, S. Castillo, M. Moran-Pineda, Journal of Alloys and Compounds., 483, (2009), 418.

[13] O. Jessensky, F. Müller, U. Gösele, Journal of the Electrochemical Society., 145, (1998), 3735 .

[14] K. Biswas, H. Matbouly, V. Rawat, J. Schroeder, T. Sands, Applied Physics Letters., 95 (2009), 073108.

[15] P. Bocchetta, C. Sunseri, G. Chiavarotti, F. Di Quarto, Electrochimica Acta., 48, (2003), 3175

[16 ] C.U. Yu, C.C. Hu, A. Bai, Y.F. Yang, Pore-size dependence of AAO films on surface roughness of Al-1050 sheets controlled by electropolishing coupled with fractional factorial design, Surface and Coating Technology., 201, (2007), 7259. 